\title{
Memory effect in melting behaviour, crystallization kinetics and morphology of poly(propylene terephthalate)
}

\author{
Laura Sisti, Lara Finelli, Nadia Lotti *, Corrado Berti, Andrea Munari
}

Dipartimento di Chimica Applicata e Scienza dei Materiali, Università di Bologna, Viale Risorgimento 2, 40136 Bologna, Italy; Fax ++390512093220;

nadia.lotti@mail.ing.unibo.it

(Received: August 4, 2003; published: October 15, 2003)

\begin{abstract}
Crystallization kinetics and melting behaviour of poly(propylene terephthalate) (PPT) were investigated by means of differential scanning calorimetry and hot-stage optical microscopy. Isothermal crystallization kinetics was analysed according to the Avrami treatment. The effects of temperature and duration of melting on the overall rate of isothermal crystallization were studied: the rate was found to decrease with increasing melting temperature and melting time. This result was discussed on the basis of the gradual destruction of predetermined athermal nuclei. Values of the Avrami exponent close to 3 were obtained, regardless of the adopted thermal treatment and the crystallization temperature, $T_{c}$, in agreement with a crystallization process originating from predetermined nuclei and characterized by three-dimensional spherulitic growth. As a matter of fact, spacefilling spherulites were observed by optical microscopy at all $T_{\mathrm{c}}$ 's, independent of the applied thermal treatments. For each of them, the rate of crystallization became lower as $T_{\mathrm{c}}$ increased, as usual at low undercooling where the crystallization process is controlled by nucleation. The observed multiple endotherms, which are commonly displayed by polyesters, were influenced by $T_{\mathrm{c}}$ and ascribed to melting and recrystallization processes. Linear and non-linear treatments were applied in order to estimate the equilibrium melting temperature for PPT, by using the corrected melting temperatures. The non-linear estimation yielded an about $33^{\circ} \mathrm{C}$ higher value with respect to the one obtained by means of the linear approach. Through the analysis of secondary nucleation theory, the classical II $\rightarrow$ III transition was found to occur at a temperature of $194^{\circ} \mathrm{C}$. The average work of chain folding for nucleation was determined to be c. $5.2 \mathrm{kcal} / \mathrm{mol}$. The heat of fusion was correlated to the specific heat increment for samples with different degree of crystallinity and the results were interpreted on the basis of the existence of an interphase, whose amount was found to depend on the thermal treatment the polymer was subjected to.
\end{abstract}

\section{Introduction}

It is well known that the morphological structure (size, shape, perfection, orientation of crystallites), which is formed by crystallization from the molten state, influences strongly the properties of polymers. Thus, crystallization behaviour represents an interesting research subject in order to optimise process conditions and control the properties of the final products. However, it has to be emphasized that the study of the crystallization phenomenon of a polymer should be carried out eliminating the influence of impurities, additives, nucleating agents and crystalline memory. In fact, if 
the melting of a polymer is carried out at a temperature that does not completely destroy the crystalline memory, traces of crystalline species may persist in the melt, and upon subsequent cooling may nucleate and enhance the crystallization rate [1]. In particular, the memory effect can be erased by melting the polymer at a sufficiently high temperature, for example, above its equilibrium melting temperature $\left(T_{\mathrm{m}}{ }^{\circ}\right)$.

Poly(propylene terephthalate) (PPT) belongs to a series of engineering thermoplastic aromatic polyesters, including poly(ethylene terephthalate) (PET) and poly(butylene terephthalate) (PBT). Although the synthesis of PPT by polycondensation reaction of terephthalic acid and 1,3-propanediol was reported already in the 1950s, a recent new development in the preparation of the monomer 1,3-propanediol, by using hydroformylation of ethylene oxide, has been the key for launching its successful commercialization [2].

Several reports have appeared in the literature on the melting and crystallization behaviour of PPT. In the paper of Bulkin et al. [3], the crystallization kinetics of poly(propylene terephthalate) was investigated from the glassy state by means of rapid scanning Raman spectroscopy and FT-IR spectroscopy. Chisholm et al. [4] compared the crystallization kinetics of PET, PPT and PBT from the melt under isothermal conditions and investigated the effect of melt-mixing and of the presence of common nucleating agents on the crystallization rates. Huang et al. [5] studied the isothermal crystallization kinetics using both differential scanning calorimetry and polarized optical microscopy. Hong et al. [6] focused their attention on the correlations between crystallization behaviour and morphological changes of PPT spherulites. Up to now, no papers have appeared in the literature dealing with the influence of the memory effect on the crystallization kinetics and morphology of PPT.

It has to be emphasized that in recent years the study of the rigid-amorphous phase present in some polymers has aroused a growing interest. Generally, the highly ordered structure of most semicrystalline polymers cannot be simply described by means of a two-phase model, consisting of crystalline and amorphous phases. As a consequence, a third phase, the so-called 'rigid amorphous phase' (RAP) or 'interphase' between crystalline and amorphous layers has been taken into consideration in the structure of semi-crystalline polymers. Several polymers, such as poly(phenylene sulfide) [7,8], poly(ethylene terephthalate) [9], poly(butylene terephthalate) [10], polycarbonate (PC) [11] and poly(ether ether ketone) (PEEK) [12,13] have been found to possess an interphase as an additional intermediate component in their structure. All the mentioned polymers are relatively rigid polymers, suggesting that the formation of an interphase can be due to their poor chain flexibility. As far as PPT is concerned, Wunderlich and co-workers $[14,15]$, investigating its thermal properties as a function of processing history, suggested that PPT posses an interphase, the amorphous phase not exhibiting a usual rubber- or liquid-like behaviour above the glass transition temperature. Moreover, the solid-state NMR results reported by Grebowicz and Chuah [16] indicate that the spin-relaxation times of PPT could not be fitted using a simple two-phase model, confirming therefore the existence of a rigid amorphous phase in this polyester. More recently, Hong and co-workers [17] investigated the effect of crystallization conditions on the formation of a RAP for PPT, finding that the rigid amorphous fraction increased both with increasing crystallization temperature and crystallization time.

In this view, herein, we report the results of an investigation on the effect of crystalline memory on isothermal crystallization characteristics of a poly(propylene terephthalate) sample synthesized in our laboratories. Moreover, differential scanning calorimetry (DSC) measurements were used in order to determine the existence of a 
rigid amorphous fraction in PPT as well as the effect of thermal treatments on the RAP formation.

\section{Experimental part}

\section{Materials}

Poly(propylene terephthalate) was synthesized according to the well-known twostage polycondensation procedure, as previously reported [18], starting from dimethyl terephthalate and 1,3-propanediol, using titanium tetrabutoxide $\left(\mathrm{Ti}(\mathrm{OBu})_{4}\right)$ as catalyst. As expected, the relatively high temperature adopted and the use of $\mathrm{Ti}(\mathrm{OBu})_{4}$ lead to a polymer with a random distribution of molecular weights. The monomeric unit is:

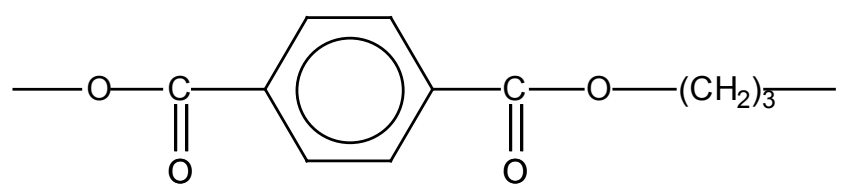

The polymer, after purification by dissolution in 1,1,1,3,3,3-hexafluoro-2-propanol and precipitation in methanol, was previously characterized by some of us from the molecular and thermal point of view [18]. The chemical structure was confirmed by means of ${ }^{1} \mathrm{H} N M R$, and the number-average molecular weight, determined with gel permeation chromatography (GPC), turned out to be 34 500. At room temperature the polymer appears as a semicrystalline solid: the calorimetric trace, obtained after rapid cooling from the melt, showed a glass transition $\left(T_{\mathrm{g}}=52^{\circ} \mathrm{C}\right)$, followed by an exothermal 'cold crystallization' peak $\left(T_{\mathrm{c}}=72^{\circ} \mathrm{C}\right)$ and a melting endotherm at higher temperature $\left(T_{\mathrm{m}}=226^{\circ} \mathrm{C}\right)$. The enthalpy associated with the crystallization exotherm does not compare with the corresponding heat of fusion, indicating that the polymer cannot be completely vitrified into the amorphous state by quenching, its crystallization rate being higher than the cooling rate [18].

\section{Calorimetric measurements}

The isothermal crystallization behaviour of PPT was investigated by using a PerkinElmer DSC7 calorimeter. The external block temperature control was set at $-60^{\circ} \mathrm{C}$. All the measurements were carried out under a nitrogen atmosphere to minimize the oxidative degradation. The instrument was calibrated with high-purity standards (indium and cyclohexane) for melting temperature and heat of fusion. The consistency of the temperature calibration was checked for each run to ensure reliability of the data obtained. To make sure that the thermal lag between the polymer sample and the DSC sensors was kept to a minimum, each sample holder was loaded with a small amount, weighing about $5 \mathrm{mg}$. It is noteworthy that each sample was used only once.

The following experimental procedure was adopted: the sample was heated up to a specified melting temperature, from 250 to $270^{\circ} \mathrm{C}$, and held there for a time ranging from 1 to $5 \mathrm{~min}$, then rapidly cooled by liquid nitrogen to the predetermined crystallization temperature $T_{\mathrm{c}}$. The $T_{\mathrm{c}}$ range was chosen in order to avoid crystallization on the cooling step and to obtain crystallization times no longer than $60 \mathrm{~min}$.

The heat flow evolving during isothermal crystallization was recorded as a function of time and the completion of the crystallization process was detected by the levelling of 
the DSC trace. For a better definition of the starting time $\left(t_{\text {start }}\right)$, for each isothermal scan a blank run was also performed with the same sample, at a temperature above the melting point, where no phase change occurred [19]. The blank run was subtracted from the isothermal crystallization scan and the start of the process was taken as the intersection of the extrapolated baseline and the resulting exothermal curve. The isothermally crystallized samples were then heated directly from $T_{\mathrm{c}}$ up to melting at $10^{\circ} \mathrm{C} / \mathrm{min}$. The melting temperature $\left(T_{\mathrm{m}}\right)$ was taken as the peak value of the endothermic phenomenon of the DSC curve. Analysis of the crystallized PPT samples by GPC confirmed that the residence at high melting temperatures for extended periods of time did not adversely affect their molar mass distribution, thereby suggesting that neither degradation processes nor post-polymerization reactions, if present, were significant.

In order to gain information about the presence of a rigid-amorphous phase in the PPT under investigation, samples characterized by different crystal/amorphous phase ratio were investigated; these were obtained subjecting the PPT polyester to the following thermal treatments:

1. the samples were partially molten in DSC by heating to various temperatures in the melting range, quickly cooled below the glass transition temperature, and reheated at $20^{\circ} \mathrm{C} / \mathrm{min}$;

2. the samples were heated to $260^{\circ} \mathrm{C}$, kept there for $1 \mathrm{~min}$ and then quenched outside the calorimeter by immersing in liquid nitrogen with different speed of transfer below the glass transition temperature, and reheated at $20^{\circ} \mathrm{C} / \mathrm{min}$.

The different thermal treatments will be described in the text where needed.

The specific heat increment, $\Delta c_{p}$, associated with the glass transition of the amorphous phase, was calculated from the vertical distance between the two extrapolated baselines at the glass transition temperature. The heat of fusion of the crystal phase was calculated from the difference between the enthalpy associated with the melting endotherm and the cold-crystallization exotherm whenever present.

\section{Optical crystallization measurements}

The isothermal rate of crystallization was also measured through the observation of the spherulitic radial growth, using a Zeiss Axioscop 2 optical polarizing microscope, equipped with a Linkam TMS94 hotstage. Isothermal crystallization measurements were performed on a small fragment of polymer, inserted between two microscope cover glasses, and subjected to the following thermal program. The sample was heated at $20^{\circ} \mathrm{C} / \mathrm{min}$ up to a temperature ranging from 250 to $270^{\circ} \mathrm{C}$, held there for different times, from 1 to 5 min (where the melt was squeezed into a film through a small pressure applied to the upper glass), then quenched by means of $\mathrm{N}_{2}$ gas flow (cooling rate $>250^{\circ} \mathrm{C} / \mathrm{min}$ ) to the selected crystallization temperature $T_{\mathrm{c}}$, where isothermal crystallization was carried out. The whole procedure was performed without removing the sample from the hot stage. A video camera attached to the microscope and connected with a computer permitted image acquisition; the crystallization process was recorded by means of Axiovision software (Zeiss) and spherulite dimensions were measured after calibration with a micrometric reticule. A new sample was used for each crystallization measurement; typically, the growth of four different spherulites was monitored at each $T_{\mathrm{c}}$. 


\section{Results and discussion}

\section{Isothermal crystallization kinetics}

The analysis of isothermal bulk crystallization kinetics is often performed using the Avrami equation concerning phase transformation [20], which is usually written in the form:

$X_{t}=1-\exp \left[-k_{n}\left(t-t_{\text {start }}\right)^{n}\right]$

where $X_{t}$ is the fraction of polymer crystallized at time $t, k_{n}$ the overall kinetic constant, $t$ is the time of the isothermal step measured from the achievement of the temperature control, $t_{\text {start }}$ the initial time of the crystallization process, as described in the Exptl. part, and $n$ the Avrami exponent, which is correlated with the nucleation mechanism and the morphology of the growing crystallites. $X_{t}$ can be calculated as the ratio between the area of the exothermic peak at time $t$ and the total measured area of crystallization. In Fig. 1a, as an example, the variations are reported of $X_{t}$ with time at various crystallization temperatures for a PPT sample molten at $260^{\circ} \mathrm{C}$ and kept at this temperature for $3 \mathrm{~min}$ : as can be seen, all the curves have a sigmoidal shape, typical of polymer crystallization behaviour. It is likewise worth remembering that Eq. (1) is usually applied to experimental data in the linearized form, by plotting $\ln \left(-\ln \left(1-X_{t}\right)\right.$ as a function of $\ln \left(t-t_{\text {start }}\right)$, permitting the determination of $n$ and $k_{n}$ from the slope and the intercept, respectively. In Fig. 1b, the linearized Avrami plots are shown for a selected set of crystallization temperatures. The presence in the curves of two zones with different slopes is evident: $\ln \left(-\ln \left(1-X_{t}\right)\right.$ varies linearly with a higher slope at an early stage and with a lower one at a later stage. This trend is usually observed in the case of polymers and attributed to a primary crystallization followed by a secondary crystallization process [20]. An analogous trend has been found for PPT samples molten at different temperatures and kept there for different times.
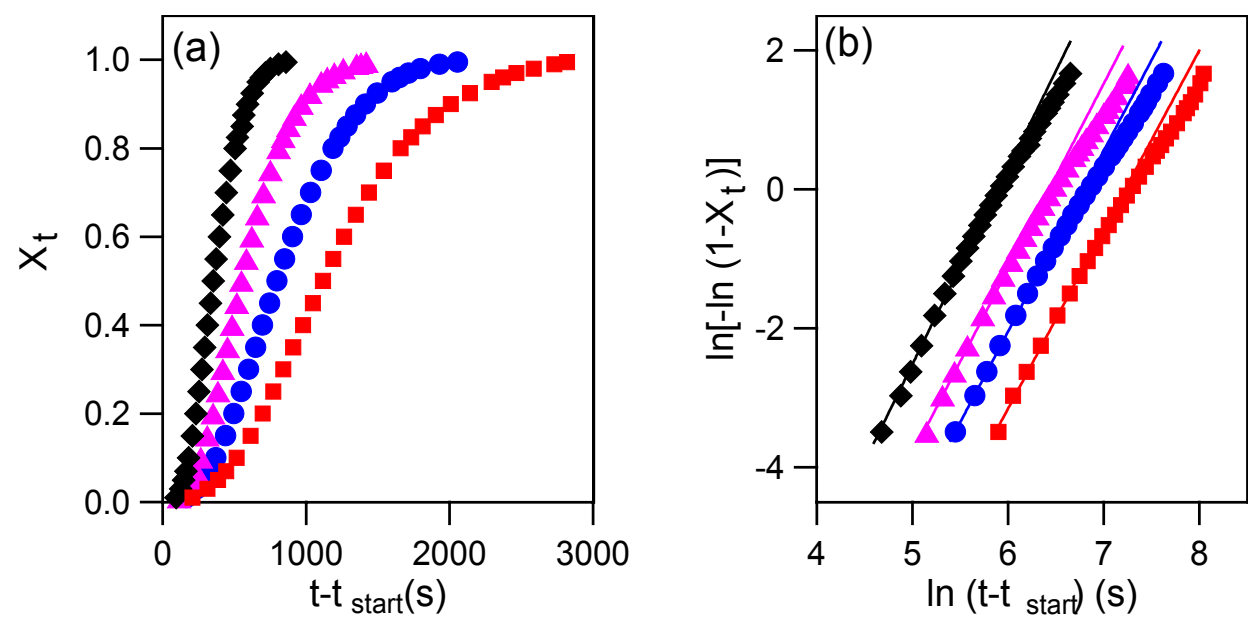

Fig. 1. (a) Crystallization isotherms and (b) linearized Avrami plots at various $T_{\mathrm{c}}$ 's: $(\bullet) 196^{\circ} \mathrm{C},(\Delta) 199^{\circ} \mathrm{C},(\bullet) 202^{\circ} \mathrm{C},($ ( $) 205^{\circ} \mathrm{C}$

The value of the kinetic constant $k_{n}$ can be obtained also by means of the following relationship:

$k_{n}=\ln 2 / t_{1 / 2}{ }^{n}$

where $t_{1 / 2}$ is the crystallization half time, defined as the time required to reach $X_{t}=$ 0.5 . 
Tab. 1. Kinetic parameters for the isothermal crystallization of PPT

\begin{tabular}{|c|c|c|c|c|}
\hline Thermal treatment & $T_{\mathrm{C}}$ in ${ }^{\circ} \mathrm{C}$ & $t_{1 / 2}$ in $\mathrm{s}$ & $n$ & $k_{n}$ in $\mathrm{s}^{-n}$ \\
\hline \multirow{8}{*}{$3 \mathrm{~min}$ at $250^{\circ} \mathrm{C}$} & 190 & 80 & 2.8 & $3.4 \cdot 10^{-6}$ \\
\hline & 193 & 111 & 2.8 & $2.3 \cdot 10^{-6}$ \\
\hline & 196 & 144 & 3.0 & $5.2 \cdot 10^{-7}$ \\
\hline & 199 & 215 & 2.9 & $7.9 \cdot 10^{-8}$ \\
\hline & 202 & 264 & 2.8 & $3.1 \cdot 10^{-8}$ \\
\hline & 205 & 447 & 2.9 & $8.4 \cdot 10^{-9}$ \\
\hline & 208 & 668 & 3.0 & $3.0 \cdot 10^{-9}$ \\
\hline & 211 & 1205 & 2.9 & $4.5 \cdot 10^{-10}$ \\
\hline \multirow{8}{*}{$3 \mathrm{~min}$ at $260^{\circ} \mathrm{C}$} & 190 & 109 & 3.0 & $1.1 \cdot 10^{-6}$ \\
\hline & 193 & 170 & 2.8 & $6.8 \cdot 10^{-7}$ \\
\hline & 196 & 206 & 2.9 & $9.0 \cdot 10^{-8}$ \\
\hline & 199 & 278 & 2.8 & $3.8 \cdot 10^{-8}$ \\
\hline & 202 & 406 & 2.8 & $1.1 \cdot 10^{-8}$ \\
\hline & 205 & 848 & 3.1 & $3.0 \cdot 10^{-9}$ \\
\hline & 208 & 1182 & 2.9 & $4.8 \cdot 10^{-10}$ \\
\hline & 190 & 156 & 3.0 & $2.0 \cdot 10^{-7}$ \\
\hline \multirow{5}{*}{$1 \mathrm{~min}$ at $270^{\circ} \mathrm{C}$} & 193 & 203 & 3.0 & $8.4 \cdot 10^{-8}$ \\
\hline & 196 & 266 & 2.9 & $3.7 \cdot 10^{-8}$ \\
\hline & 199 & 436 & 3.0 & $7.9 \cdot 10^{-9}$ \\
\hline & 202 & 734 & 3.0 & $1.6 \cdot 10^{-9}$ \\
\hline & 205 & 958 & 2.9 & $8.0 \cdot 10^{-10}$ \\
\hline \multirow{7}{*}{$3 \mathrm{~min}$ at $270^{\circ} \mathrm{C}$} & 190 & 242 & 2.8 & $5.1 \cdot 10^{-08}$ \\
\hline & 193 & 297 & 3.0 & $2.6 \cdot 10^{-08}$ \\
\hline & 196 & 389 & 2.9 & $1.1 \cdot 10^{-08}$ \\
\hline & 199 & 630 & 2.9 & $2.7 \cdot 10^{-09}$ \\
\hline & 202 & 938 & 3.0 & $8.3 \cdot 10^{-10}$ \\
\hline & 205 & 1178 & 2.8 & $4.2 \cdot 10^{-10}$ \\
\hline & 190 & 322 & 2.8 & $2.1 \cdot 10^{-08}$ \\
\hline \multirow{4}{*}{$5 \mathrm{~min}$ at $270^{\circ} \mathrm{C}$} & 193 & 388 & 3.0 & $1.3 \cdot 10^{-08}$ \\
\hline & 196 & 576 & 2.9 & $3.5 \cdot 10^{-09}$ \\
\hline & 199 & 746 & 2.9 & $1.7 \cdot 10^{-09}$ \\
\hline & 202 & 1534 & 3.0 & $2.0 \cdot 10^{-10}$ \\
\hline \multirow{6}{*}{$\begin{array}{c}3 \mathrm{~min} \text { at } 250^{\circ} \mathrm{C} \\
\text { (twice treated sample) }\end{array}$} & 190 & 215 & 2.8 . & $6.9 \cdot 10^{-08}$ \\
\hline & 193 & 290 & 2.9 & $2.8 \cdot 10^{-08}$ \\
\hline & 196 & 432 & 2.8 & $8.6 \cdot 10^{-09}$ \\
\hline & 199 & 581 & 3.0 & $3.5 \cdot 10^{-09}$ \\
\hline & 202 & 875 & 2.9 & $1.2 \cdot 10^{-09}$ \\
\hline & 205 & 1900 & 2.8 & $1.0 \cdot 10^{-10}$ \\
\hline
\end{tabular}


The parameter $n$, the kinetic constants $k_{n}$ and $t_{1 / 2}$ are collected in Tab. 1. Examining the data relative to each thermal treatment applied to the sample, one can observe that the overall kinetic constant $k_{n}$ regularly decreases with increasing $T_{\mathrm{c}}$, as usually happens at low undercooling, where crystal formation is controlled by nucleation. The Avrami exponent $n$ is close to 3 for all the crystallization temperatures investigated, indicating that the crystallization process originates from predetermined nuclei and is characterized by three-dimensional spherulitic growth. Moreover, the value of the Avrami exponent appears to be the same, independent of the thermal treatment applied.

\section{Effect of final temperature}

In order to evaluate the effect of the final temperature on the crystallization rate, as an example, the half-crystallization times $t_{1 / 2}$ of samples heated at three different temperatures for a fixed time of $3 \mathrm{~min}$ were plotted as a function of $T_{\mathrm{c}}$ in Fig. 2. As can be seen, a marked increase of $t_{1 / 2}$ is observed as the adopted final temperature is increased, indicating that the half-crystallization times have a strong correlation with the final temperature used. The same trend was observed keeping the polymer at the selected final temperature for 1 or $5 \mathrm{~min}$. This result can be explained on the basis of the 'crystalline memory' of the polymer, which refers to clusters of molecules that retain their crystal structure because of insufficient temperature or holding time at the final temperature. In fact, the ones which survive at $T_{\mathrm{c}}$ can act as nuclei (provided they exceed the critical nucleus size), greatly enhancing the overall crystallization rate. In addition, the results reported in Fig. 2 can be considered as an evidence, even though only indirect, that the average number of crystalline residues that can act as predetermined athermal nucleation sites decreases with increasing value of adopted final temperature.

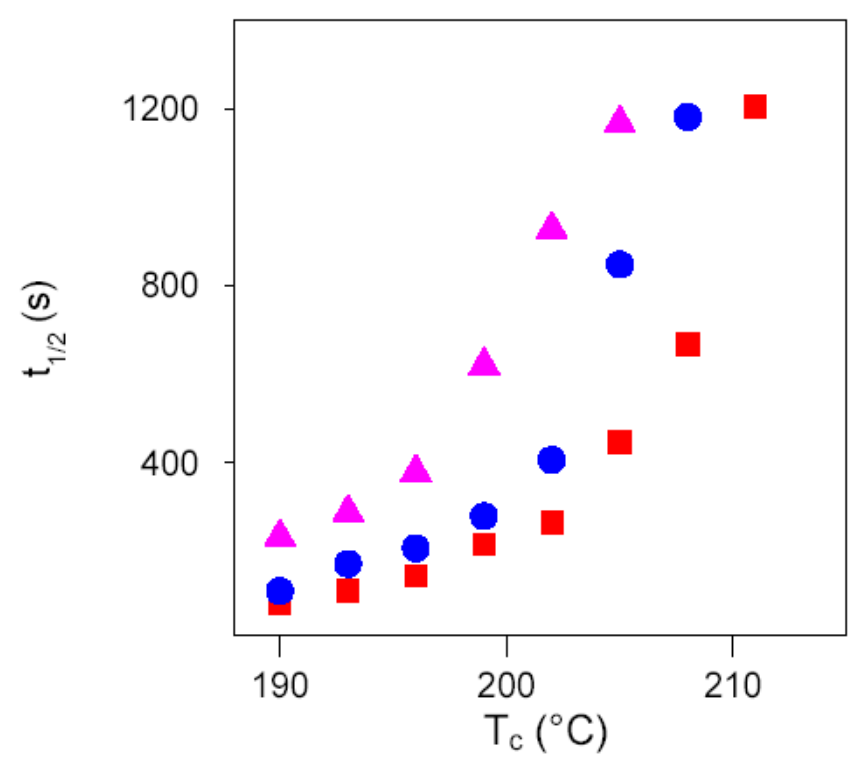

Fig. 2. Crystallization half time $\left(t_{1 / 2}\right)$ vs. $T_{\mathrm{c}}$ after $3 \mathrm{~min}$ at: $(\square) 250^{\circ} \mathrm{C} ;(\bullet) 260^{\circ} \mathrm{C}$; ( $\triangle$ ) $270^{\circ} \mathrm{C}$

\section{Effect of holding time}

In order to evaluate the effect of holding time on the crystallization rate, the halfcrystallization times $t_{1 / 2}$ of samples molten at $270^{\circ} \mathrm{C}$ for different times were plotted as 
a function of $T_{\mathrm{c}}$ in Fig. 3. As can be seen, a marked increase in $t_{1 / 2}$ is observed as the holding time increases. Consequently, for the particular value of final temperature applied, the number of nuclei decreases with increasing holding time, as suggested by Ziabicki and Alfonso [21,22], who proposed that the total concentration of predetermined nuclei is an exponential decay function of the residence time in the melt. Different results were obtained, however, when the adopted final temperatures were 250 and $260^{\circ} \mathrm{C}$, no effect of holding time on the overall crystallization rate being indeed found. Such behaviour could be attributed to too short residence times at these temperatures. In order to investigate more deeply this trend, further experiments are currently carried out and the results will be reported elsewhere.

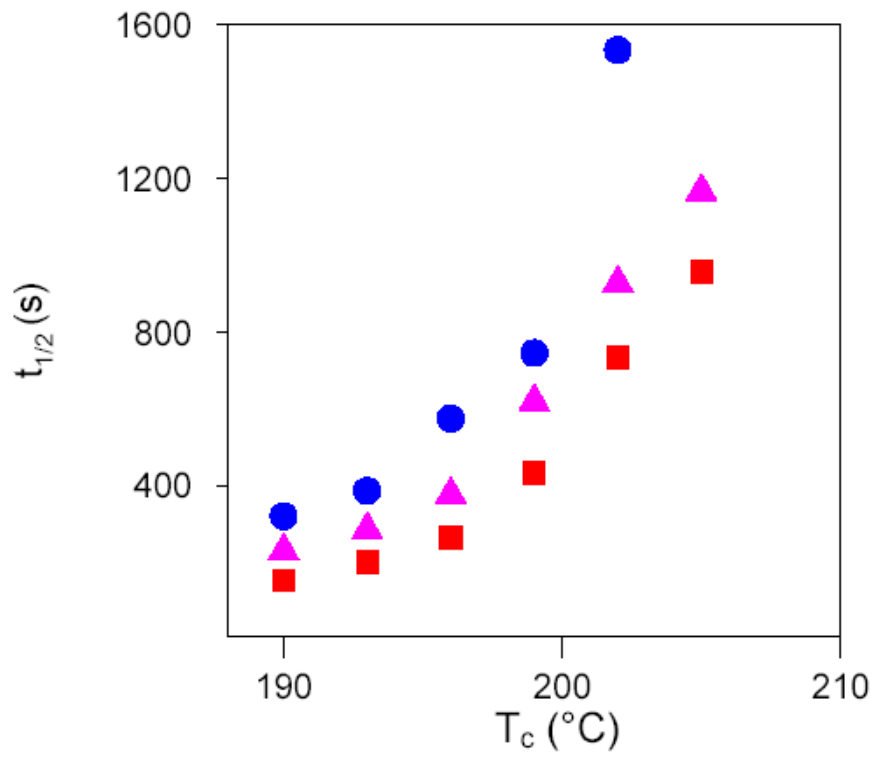

Fig. 3. Crystallization half time $\left(t_{1 / 2}\right)$ vs. $T_{\mathrm{c}}$ after different times at $270^{\circ} \mathrm{C}$ : (घ) $1 \mathrm{~min}$; $(\Delta) 3 \mathrm{~min} ;(\bullet) 5 \mathrm{~min}$

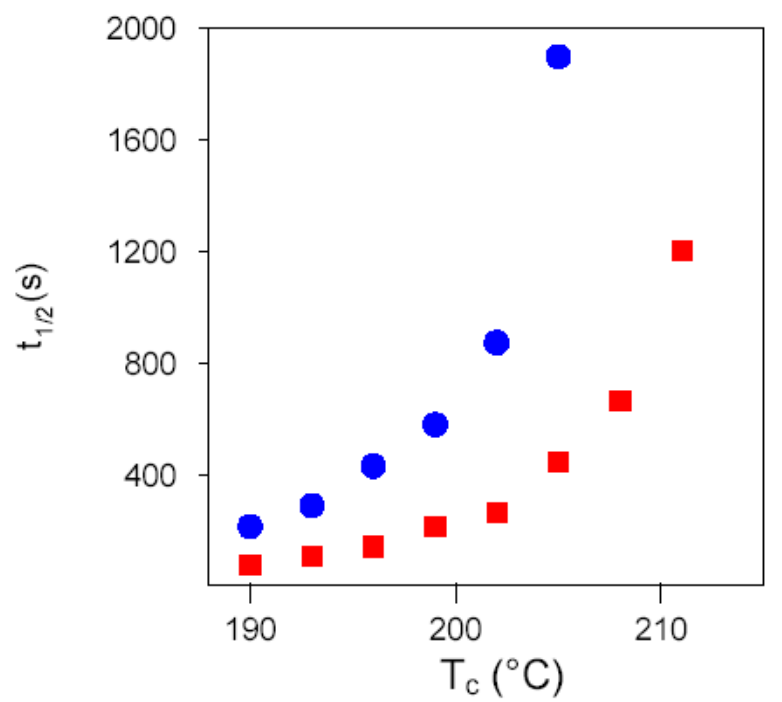

Fig. 4. Crystallization half time $\left(t_{1 / 2}\right)$ vs. $T_{\mathrm{c}}$ after $3 \mathrm{~min}$ at $250^{\circ} \mathrm{C}$ : $(\square)$ once treated sample; $(\bullet)$ twice treated sample

Further evidence that the 'crystalline memory' strongly affects the crystallization process of the polymer has been obtained subjecting the same sample of PPT to two 
identical and consecutive thermal cycles, each of one consisting in melting the polymer at $250^{\circ} \mathrm{C}$ for 3 min and then rapidly cooling at the selected crystallization temperature. The results obtained are reported in Fig. 4, where the half times of crystallization are plotted as a function of $T_{\mathrm{c}}$ : as can be seen, the $t_{1 / 2}$ values of the sample twice treated are higher than those of the once treated polymer. This behaviour can be again explained on the basis of the number of residue crystallites, which remain in the melt and during cooling form the predetermined nuclei of crystallization: this amount becomes the lower the higher the number of thermal cycles the polymer is subjected to.

In order to confirm the hypothesis that the traces of crystalline species, which probably persist in the melt and upon subsequent cooling may nucleate, are responsible for the observed enhancement of the crystallization rate, the isothermal crystallization kinetics was also investigated by means of hot-stage optical microscopy. In fact, as well known, the spherulitic growth rate is independent of the number of nuclei present in the melt and, consequently, should not be affected by the 'memory effect', different from the overall crystallization rate, which is the result of nucleation and growth processes. The PPT samples were therefore subjected to the various thermal treatments described in the Exptl. part. In all cases, independent of the chosen thermal treatment, upon solidification from the melt, PPT adopts a spherulitic morphology (see, as an example, Fig. 5).

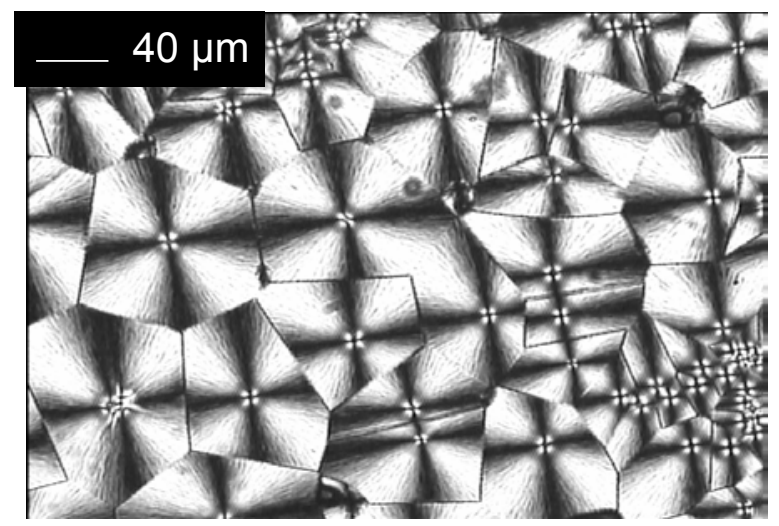

$190^{\circ} \mathrm{C}$

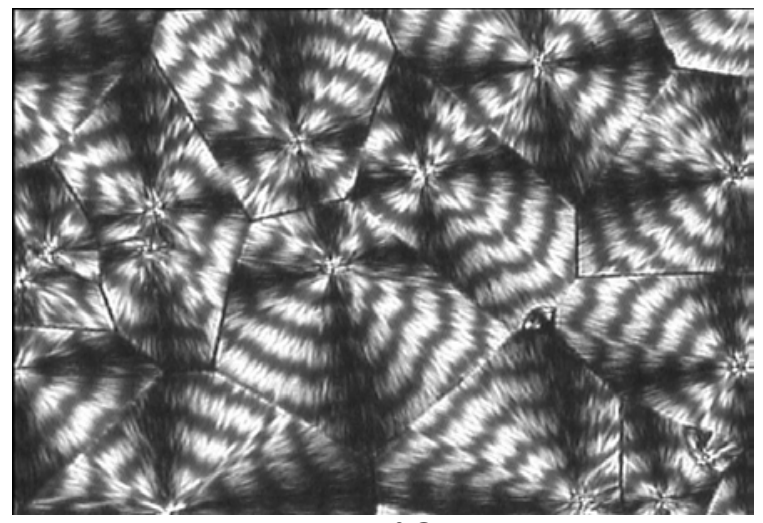

$196^{\circ} \mathrm{C}$

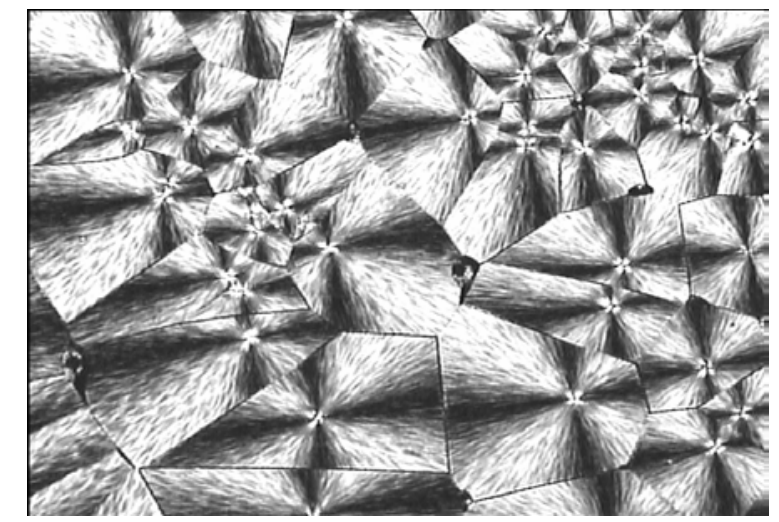

$193^{\circ} \mathrm{C}$

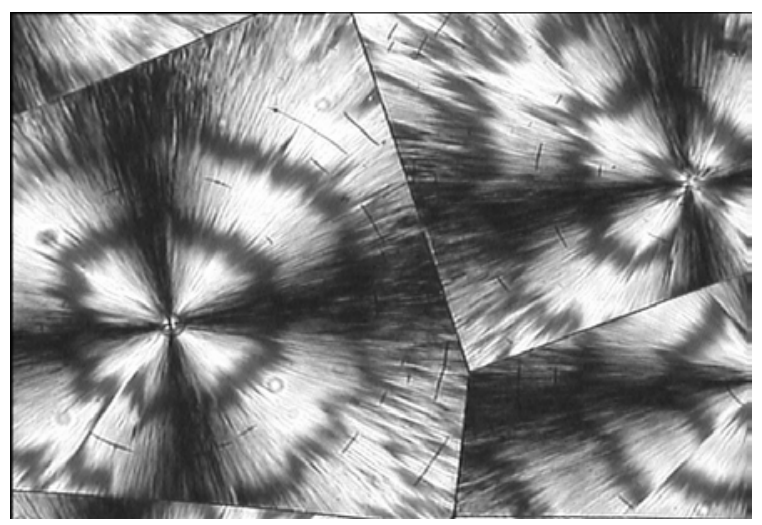

$199^{\circ} \mathrm{C}$

Fig. 5. Optical micrographs of spherulites isothermally crystallized at the indicated $T_{\mathrm{c}}$ 's

Moreover, the spherulite dimensions increase with increasing $T_{c}$, owing to a decrease of nucleation rate [23], which produces fewer spherulites that can grow larger before 
impinging. Measurements of the spherulite radius $(R)$ as a function of time yield linear plots, such as the ones reported in Fig. 6a, whose slopes, which represent the radial growth rate $G$, change with $T_{\mathrm{c}}$. As shown in Fig. 6b, where $G$ is reported as a function of $T_{\mathrm{c}}$, the rate of crystallization decreases with increasing crystallization temperature, as expected at high values of $T_{\mathrm{c}}$ (low undercooling). In fact, in these conditions, the determining step of the crystallization process is nucleation, since the diffusion rate is high at high temperature. By comparing the $G$ values of PPT samples subjected to the various thermal treatments, no dependence of spherulitic growth rate on the adopted melting temperature and holding time was indeed found. This result undoubtedly proves that the dependence of the overall crystallization rate on the applied thermal treatments is really due to subcritical crystalline aggregates, which survive in the melt for long time and behave as predetermined athermal nuclei.

As far as the morphology of the crystalline phase is concerned, in agreement with the results reported by other authors [5,6], PPT showed spherulites characterized by a 'Maltese cross' birefringent pattern. In particular a morphological transition from nonbanded spherulites to banded ones was observed with increasing $T_{c}$; a further increment of crystallization temperature was found to cause again the disappearance of the banded structure. Moreover, the nucleation density increases and the spherulite texture becomes finer as $T_{\mathrm{c}}$ is decreased. In the case of PPT under investigation, no accurate measurements of band spacing as a function of $T_{\mathrm{c}}$ could be carried out, probably because of the extremely small crystallite size, due to the very high nucleation density. Lastly it was observed that the morphological changes occurred at the same temperatures indicated by Hong and co-workers [6] that ascribed such changes to regime transitions.
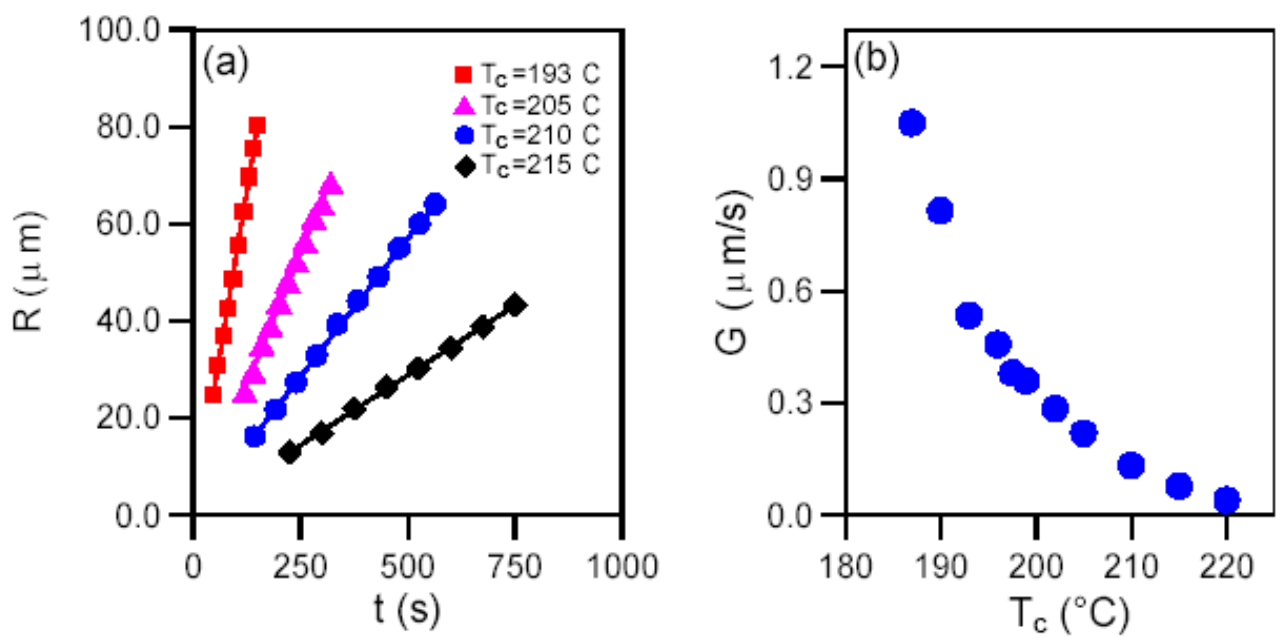

Fig. 6. Optical-microscope crystallization data: (a) time dependence of the spherulite radius at various $T_{\mathrm{c}}$ 's, (b) radial growth rate as a function of $T_{\mathrm{c}}$

\section{Melting behaviour of isothermally crystallized samples}

In order to determine the equilibrium melting temperature of PPT, it is necessary to investigate carefully the melting behaviour of the polymer. PPT was therefore isothermally crystallized from the melt, according to the various thermal treatments described in the Exptl. part, between 190 and $205^{\circ} \mathrm{C}$, with an increment of $3^{\circ} \mathrm{C}$. First of all, it is worth emphasizing that no effect of the thermal treatment on the melting behaviour of the polymer was found. For this reason, in the following we will refer 
exclusively to the results of one thermal treatment (melting at $260^{\circ} \mathrm{C}$ for $3 \mathrm{~min}$ ). Fig. 7 shows typical calorimetric traces of PPT samples isothermally crystallized at various temperatures $\left(T_{\mathrm{c}}\right)$. As can be seen, the DSC curves exhibit two endotherms, whose peaks have been labelled with Roman numbers (I and II) in the order of increasing temperature. An analogous behaviour was previously found by Chisholm et al. [4], Huang et al. [5] and Wu et al. [24] It is well known that a lot of semicrystalline polymers as well as their copolymers show multiple endothermic peaks [7-12]. Some authors associate the peaks with two distinct crystal populations [25]; others relate them to a recrystallization process occurring during the DSC scan $[19,26]$. In addition, both processes could operate at different undercooling degrees [27]. The multipleendotherm behaviour is typical of many polyesters, for the best-studied of which the phenomenon has often been ascribed to a reorganization process taking place during the DSC scan, due to a mechanism based on melting and recrystallization of less perfect crystallites into thicker crystals, followed by a final melting process at higher temperature $[19,26]$.

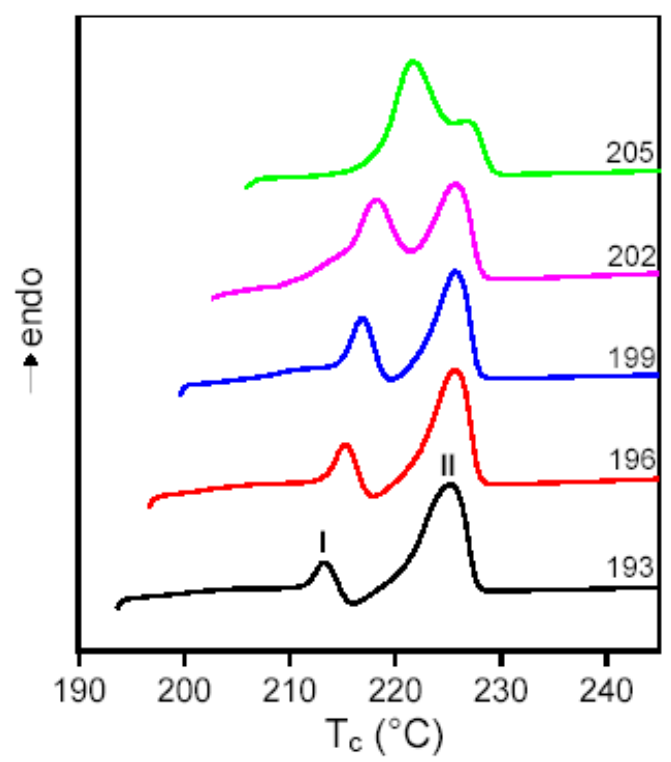

Fig. 7. DSC melting endotherms after isothermal crystallization at the indicated $T_{\mathrm{c}}$ 's, (heating rate: $10^{\circ} \mathrm{C} / \mathrm{min}$ )

As concerns PPT, the lowest-temperature endotherm (peak I) has to be ascribed to the fusion of the crystals grown by normal primary crystallization during the isothermal period at $T_{\mathrm{c}}$. It exhibits a strong dependence on the crystallization temperature, in terms of both peak position and area, i.e., the endotherm is detected at higher temperatures and its area progressively increases as $T_{\mathrm{c}}$ is raised. Such an increase suggests that thicker crystalline lamellae develop with increasing $T_{c}$. Endotherm I is followed by an exothermic peak, whose intensity regularly decreases with increasing $T_{\mathrm{c}}$, the crystallites being more and more perfect as the crystallization temperature is risen. On the contrary, the location of the highest-temperature melting endotherm (II), whose intensity decreases with increasing $T_{\mathrm{c}}$, shows no dependence on the crystallization temperature. Therefore, this last peak can be attributed to the melting of crystals grown during the heating run. In order to further investigate the nature of the double endotherms, the effect of the scanning rate on the melting behaviour of PPT samples has been analysed (see Fig. 8). It can be observed that the magnitude of melting peak I increases as the heating rate is increased, contrarily 
to the high-temperature melting peak II, whose intensity regularly decreases with the heating rate. The increasing value of the heat of fusion of melting peak I, as the heating rate is increased, indicates that the crystals formed at $T_{\mathrm{c}}$ don't have enough time to melt and recrystallize, confirming therefore a mechanism based on melting and recrystallization of less perfect crystallites into thicker crystals, which melt at higher temperature. Chisholm et al. [4], Huang et al. [5] and Wu et al. [24] came to the same conclusions, investigating the multiple melting behaviour of PPT.

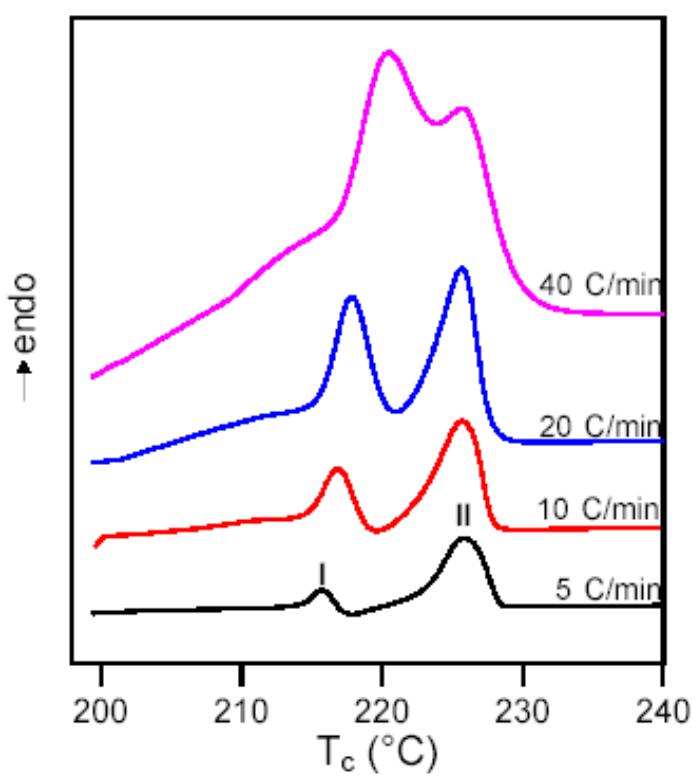

Fig. 8. DSC melting endotherms of PPT scanned at the indicated heating rate after isothermal crystallization at $199^{\circ} \mathrm{C}$. The curves have not been corrected for the changes in the instrumental signal with heating rate

However, an accurate determination of the equilibrium melting temperature requires the correction of the melting temperatures, their determination being characterized by a significant error when endo- and exothermal phenomena take place quasi simultaneously. In addition, thermal lag of the DSC cell inevitably causes the melting peak to elevate to a higher temperature, especially when a high-resistance sample, such as a polymer, is analysed. In order to overcome these disadvantages, a correction of the effect of thermal lag and a calorimetric study using various heating rates have been carried out, similarly to what was previously done by other authors [24,28]. As well known, the increment in the observed peak temperature is proportional to the square root of the heating rate at constant thermal resistance, heat of fusion and sample weight, on the basis of the theory of heat-flow calorimetry.

The observed melting temperatures therefore have been plotted as a function of the square root of heating rate, $\beta^{1 / 2}$, for the different $T_{\mathrm{c}}$ 's (see some examples for endotherm I in Fig. 9). In this procedure, the true melting temperatures can indeed be obtained by linear extrapolation to zero heating rate.

One of the most commonly used procedures to determine the equilibrium melting temperature of a polymer $\left(T_{\mathrm{m}}{ }^{\circ}\right)$ is the Hoffman-Weeks method. The popularity of this approach is due to its simplicity, needing only the experimental melting temperature of the crystallites formed at $T_{\mathrm{c}}$, which are correlated to the corresponding crystallization temperature by the following equation [29]: 
where $\gamma$ is a factor, which depends on the lamellar thickness. More precisely, $\gamma=1 / I^{*}$, where $I$ and $I^{*}$ are the thickness of the grown crystallite and of the critical crystalline nucleus, respectively [29]. Moreover, if the thickening process is fast, it is necessary [29] to investigate samples with low levels of crystallinity. Consequently PPT samples were quenched from the melt to the desired crystallization temperature and maintained at $T_{\mathrm{c}}$ until the crystallization had proceeded to $10 \%$ of the overall process.

In this view, the corrected melting temperatures are reported in Fig. 10a as a function of $T_{\mathrm{c}}$. For comparison, the uncorrected melting points (i.e., measured at $10^{\circ} \mathrm{C} / \mathrm{min}$ ) were also plotted in the same figure.

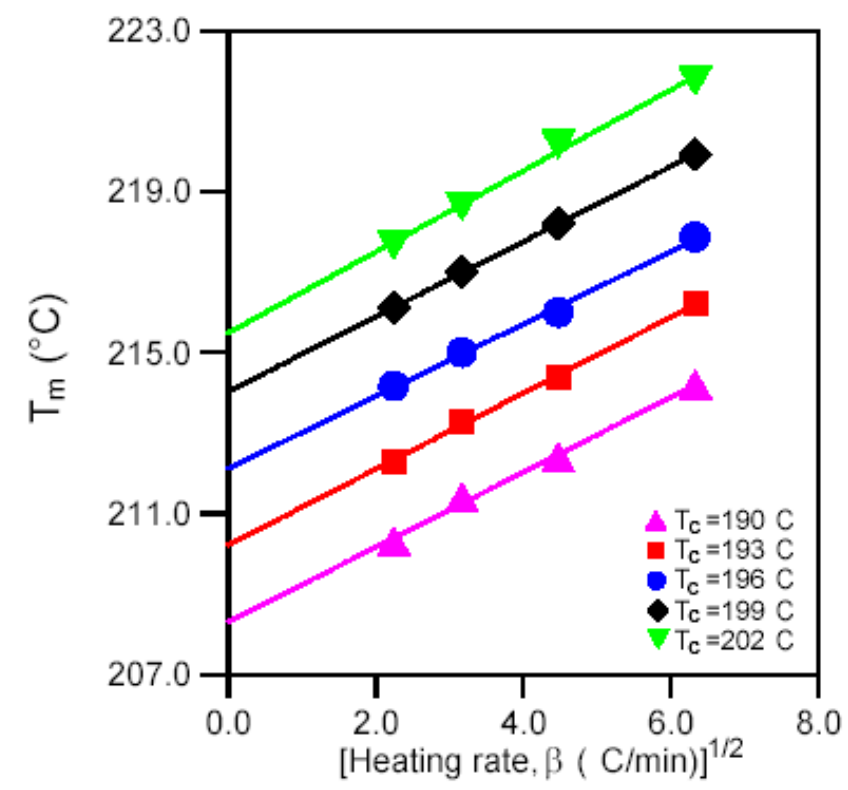

Fig. 9. Effect of the square root of heating rate on the location of the endotherm of PPT
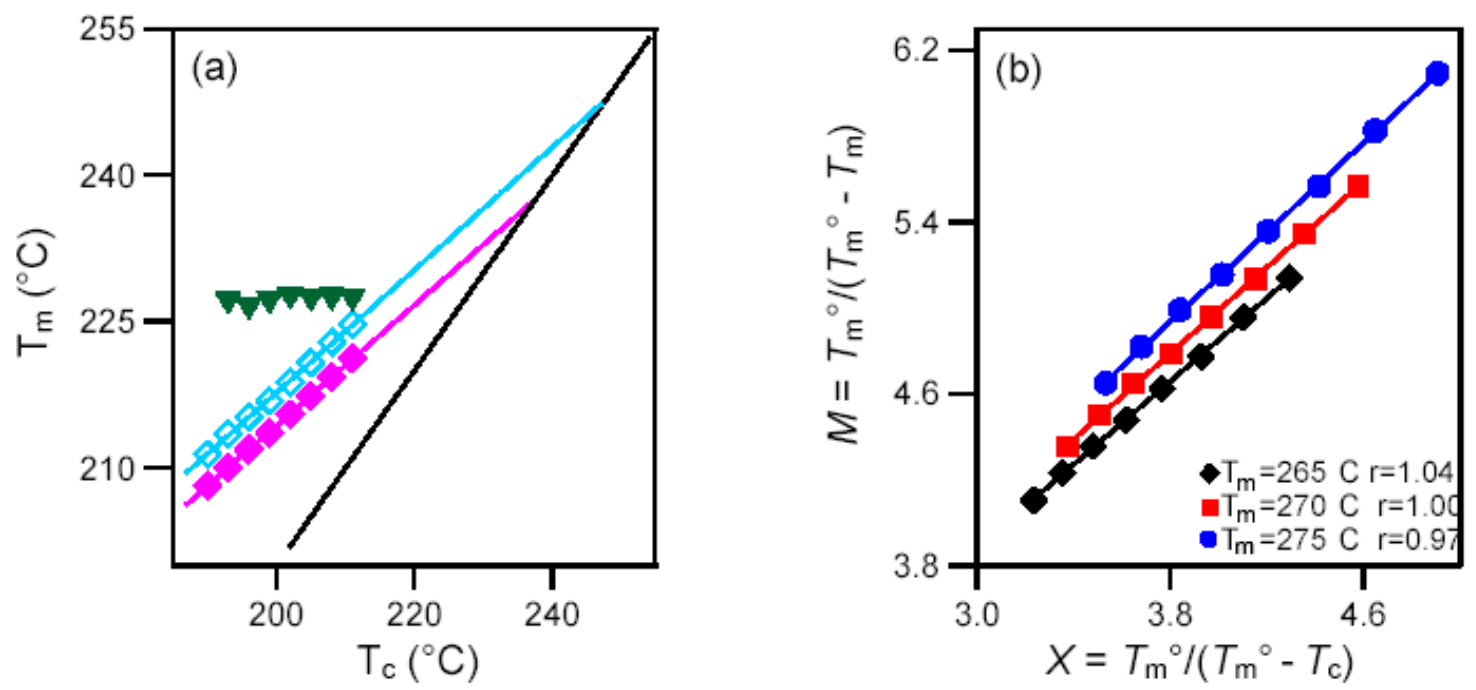

Fig. 10. (a) Hoffman-Weeks extrapolation plot of $T_{\mathrm{m}}$ vs. $T_{\mathrm{c}}$ : open symbols are uncorrected $T_{\mathrm{m}}$ and full symbols corrected $T_{\mathrm{m}}$. (b) Plot of the scaled observed melting temperature $\left(M=T_{\mathrm{m}}{ }^{\circ} /\left(T_{\mathrm{m}}{ }^{\circ}-T_{\mathrm{m}}\right)\right)$ versus the scaled crystallization temperature $(X=$ $\left.T_{\mathrm{m}}{ }^{\circ} /\left(T_{\mathrm{m}}{ }^{\circ}-T_{\mathrm{c}}\right)\right)$ for different values of the equilibrium melting temperature 
First of all, it has to be emphasized that corrected and uncorrected values concerning endotherm II are practically identical. As concerns endotherm I, it is clearly related to the original main crystal population and its location reflects the higher perfection of the crystals grown at higher temperatures. On the contrary, the melting endotherm II is observed at a rather constant temperature, characteristic of the material partially recrystallized into a more stable form on heating. In Fig. 10a, the linear extrapolation of the experimental data up to the $T_{\mathrm{m}}=T_{\mathrm{c}}$ line is also drawn, and $T_{\mathrm{m}}{ }^{\circ}$ values of $237^{\circ} \mathrm{C}$ and $247^{\circ} \mathrm{C}$ were obtained by using the corrected and uncorrected melting points, respectively. Recently, Marand and co-workers [30,31] discussed the validity of the assumption which represents the basic premise of the linear Hoffmann-Weeks treatment, i.e., the independence of the thickening coefficient for lamellae, $\gamma$, of $T_{\mathrm{c}}$ and time. As demonstrated by some results appeared in the literature [24,30-32], the linear extrapolation, even when carried out for lamellar crystals exhibiting a constant $\gamma$ value, invariably underestimates $T_{\mathrm{m}}{ }^{\circ}$ and leads to an overestimation of the $\gamma$ value. Therefore, Marand and co-workers [30,31] proposed a non-linear Hoffmann-Weeks procedure to estimate $T_{\mathrm{m}}{ }^{\circ}$, which consists in plotting the scaled melting temperature $M=T_{m}{ }^{\circ} /\left(T_{m}{ }^{\circ}-T_{m}\right)$ vs. the scaled crystallization temperature $X=T_{m}{ }^{\circ} /\left(T_{m}{ }^{\circ}-T_{c}\right)$ for various choices of $T_{\mathrm{m}}{ }^{\circ}$. The constant slope gives the value of the lamellar-thickening coefficient. For a set of experimental $T_{\mathrm{m}}$ and $T_{\mathrm{c}}$ values, a corresponding set of $M$ and $X$ values can be calculated for a given choice of $T_{\mathrm{m}}{ }^{\circ}$. When this method is applied to the melting of 'original' crystals $(\gamma=1)$, the plot of $M$ versus $X$ for the 'true equilibrium melting temperature' yields a straight line characterised by a unity slope. Fig. 10b displays the typical $M-X$ plot for PPT crystallized at various $T_{c}$ 's, for different selected values of $T_{\mathrm{m}}^{\circ}$. The equilibrium melting temperature obtained by means of the nonlinear treatment turned out to be $270^{\circ} \mathrm{C}$, using the corrected values of $T_{\mathrm{m}}$. It is clear that the linear and non-linear extrapolation led to significant differences in the equilibrium melting temperature determination, the non-linear treatment estimate being higher by about $33^{\circ} \mathrm{C}$ as compared with the linear approach. Moreover, the equilibrium melting temperature calculated by means of the non-linear extrapolation is found to be in excellent agreement with the value obtained by Wu et co-workers [24], who also applied Marand's approach.

\section{Analysis from secondary nucleation theory}

As mentioned above, a linear increment in spherulite radius with time was observed at all the $T_{\mathrm{c}}$ 's investigated, permitting the determination of the spherulitic growth rate. According to the kinetic theory [33] of polymer crystallization, the temperature dependence of the linear growth rate $(G)$ is given by the following relationship:

$$
G=G_{0} \exp \left[\frac{-U^{*}}{R\left(T_{\mathrm{c}}-T_{\infty}\right)}\right] \exp \left[\frac{-K_{\mathrm{g}}}{T_{\mathrm{c}}(\Delta T) f}\right]
$$

where the first term in parentheses represents a contribution due to the diffusion of the polymeric segments, whereas the second concerns the thermodynamic driving force. In Eq. (4), $G_{0}$ is a pre-exponential factor which contains temperatureindependent terms, $U^{*}$ is the activation energy for the transport of crystallizable segments at the liquid-solid interphase, $T_{\infty}$ the hypothetical temperature below which viscous flow ceases, $f$ a correction factor that accounts for the change of $\Delta H_{f}^{\circ}$ (enthalpy of fusion of the perfect crystals) with temperature, being $f=2 T_{\mathrm{c}} /\left(T_{\mathrm{m}}{ }^{\circ}+T_{\mathrm{c}}\right)$, $\Delta T$ the degree of undercooling $\left(\Delta T=T_{\mathrm{m}}{ }^{\circ}-T_{\mathrm{c}}\right)$, and $K_{\mathrm{g}}$ is the nucleation constant, expressed as: 


$$
K_{\mathrm{g}}=\frac{n_{\mathrm{e}} b_{0} \sigma \sigma_{\mathrm{e}} T_{\mathrm{m}}^{0}}{\Delta H_{\mathrm{m}}^{0} k d}
$$

In Eq. (5) $n_{\mathrm{e}}$ is a parameter which depends on the regime of crystallization [33], $b$ the distance between two adjacent fold planes, $\sigma$ and $\sigma_{\mathrm{e}}$ the free energies of formation per unit area of the lateral and folding surfaces, respectively, $d$ the density and $k$ the Boltzmann constant. For the calculation of $K_{\mathrm{g}}$, the $b$ value proposed by Hong and coworkers [6] was used; this value does not correspond to the $b$ parameter of the crystal unit cell. Suzie et al. [34] have suggested that chain folding takes place along the (010) crystal plane; as a consequence, the $b$ value corresponds to the perpendicular separation of crystal growth planes [6], which is not equal to the $b$ parameter of the crystal unit cell, this latter being triclinic for PPT.

Generally, it is convenient to rewrite Eq. (4) in a logarithmic form:

$\log G+\left[\frac{-U^{*}}{2.303 \cdot R\left(T_{\mathrm{c}}-T_{\infty}\right)}\right]=\log G_{0}-\frac{K_{\mathrm{g}}}{2.303 \cdot T_{\mathrm{c}}(\Delta T) f}$

In the calculation, we didn't use for $U^{*}$ the 'universal' value of $1500 \mathrm{cal} / \mathrm{mol}$, usually employed for most polymers: $U^{*}=2500 \mathrm{cal} / \mathrm{mol}$ was indeed chosen, as suggested by Hong and co-workers [6]. These authors obtained this value specifically for PPT by means of a common simulation method applied to their crystallization data. The plot of $\log G+2.303 R\left(T_{\mathrm{c}}-T_{\infty}\right)$ versus $10^{5} /\left(2.303 T_{\mathrm{c}} \Delta T f\right)$ is shown in Fig. 11; two linear trends can be evidenced. The result of the analysis of the full set of data through secondary nucleation theory clearly indicates the classical regime $\mathrm{II} \rightarrow \mathrm{II}$ transition at $194^{\circ} \mathrm{C}$, in agreement with the results obtained both by Huang and Chang [5] and Hong and co-workers [6]. $K_{\text {gllI }} / K_{\text {gll }}$ turned out to be 2.04 (see Tab. 3), as predicted by the Lauritzen-Hoffman theory [33].

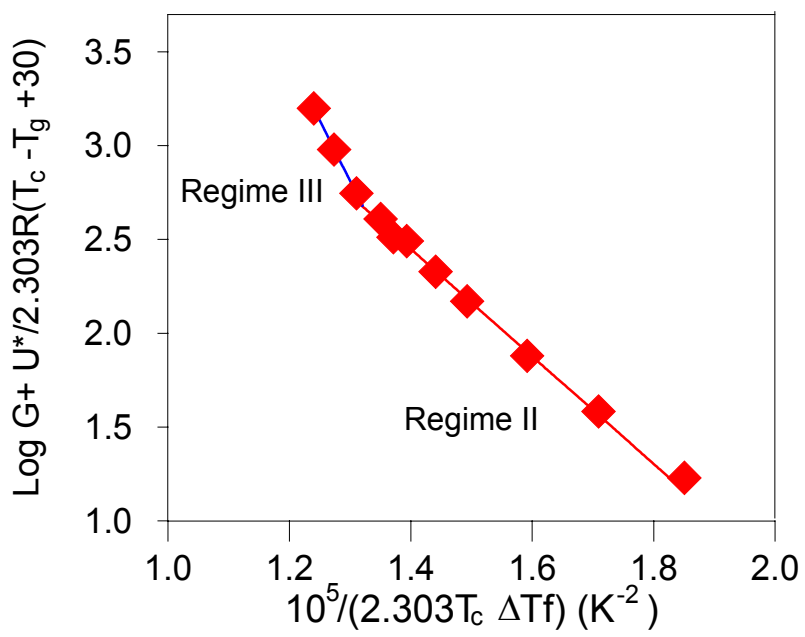

Fig. 11. Kinetic analysis of the spherulitic growth rate data (see text) for PPT samples

The $K_{\mathrm{g}}$ values were employed to calculate the product $\sigma \sigma_{\mathrm{e}}$ on the basis of Eq. (5): it turned out to be $908 \mathrm{erg} / \mathrm{cm}^{4}$, using the layer thickness $b=5.71 \AA[6], T_{\mathrm{m}}{ }^{\circ}=270^{\circ} \mathrm{C}$ and $\Delta H_{\mathrm{m}}{ }^{\circ}=30 \mathrm{~kJ} / \mathrm{mol}$ [14]. The lateral surface free energy $\sigma$ can be estimated by means of the empirical relationship proposed by Thomas and Stavely [35]:

$\sigma=\alpha \cdot \Delta H_{\mathrm{m}}^{0} \cdot \sqrt{a_{0} b_{0}}$ 
where $a_{0}$ and $b_{0}$ are the molecular width and molecular layer thickness, respectively, and $\alpha$ is an empirical constant. Generally, this latter ranges from 0.1 to 0.3 , strongly depending on the chemical structure of the polymer. In the present paper, an $\alpha$ value of 0.18 was used, as calculated specifically for PPT by Hong and co-workers [6]. The lateral surface free energy turned out to be $13.5 \mathrm{erg} / \mathrm{cm}^{2}$ and, by using Eq. (5), a fold surface-free energy $\sigma_{\mathrm{e}}$ of $67.3 \mathrm{erg} / \mathrm{cm}^{2}$ was derived.

Finally the work of chain folding $q$ can be obtained directly from the fold surface free energy by means of the relationship:

$q=2 \sigma_{\mathrm{e}} \cdot a_{0} \cdot b_{0}$

It has to be emphasized that $q$ is a parameter closely correlated with the molecular structure, i.e., the inherent stiffness of the chain itself. A value of $5.2 \mathrm{kcal} / \mathrm{mol}$ was estimated and can be considered a reasonable result, being intermediate between those of PET and PBT. A similar result was found by Hong and co-workers [6].

\section{Thermodynamic parameters}

For several of the well known semicrystalline polyesters, a rigid amorphous phase was hypothesized $[14,36,37]$. The interphase is defined as that portion of noncrystalline material, which does not mobilize at the glass transition temperature and therefore does not contribute to the observed specific heat increment. As a consequence, three distinguishable phases can be hypothesized to exist in semicrystalline polymers: (i) a crystalline phase, due to the crystallizable component, (ii) a 'normal' amorphous phase, (iii) an interphase occurring in the vicinity of the crystallites.

In order to evaluate the existence of a rigid-amorphous phase in PPT, the relationship between the specific heat increment at $T_{\mathrm{g}}$ and the heat of fusion of samples with different crystal/amorphous ratio was examined. The experimental $\Delta H_{\mathrm{m}} v s . \Delta c_{\mathrm{p}}$ data (see both full circles and full triangles), plotted in Fig. 12, show a very good linear fit: as expected, $\Delta c_{p}$ steadily decreases as the melting enthalpy (proportional to the degree of crystallinity) increases. The extrapolation to $\Delta c_{p}=0$ gives a value of 15 $\mathrm{kJ} / \mathrm{mol}$, which is much lower than the $\Delta H_{\mathrm{m}}{ }^{\circ}$ value reported in the literature by Wunderlich [14], indicating the presence of a fraction of a rigid-amorphous phase in the PPT under investigation. The calculation of the interphase fraction for the investigated samples characterized by different degrees of crystallinity has been carried out and values ranging from 10 to $45 \%$ were found. In the paper by Wunderlich et al. [14], lower rigid amorphous fractions were reported. This result is not surprising, since it is well known that the relative amounts of crystal, mobile amorphous and rigid amorphous phases depend upon the thermal treatments that the material is subjected to $[8,38]$. In fact, the thermal history affects the area of the crystallineamorphous boundary, the degree of irregularity of the crystalline phase and the molecular mobility, crucial factors for the formation and amount of a rigid-amorphous phase. In this view, some PPT samples have been subjected to drastic thermal treatments, consisting in melting the polymer at $260^{\circ} \mathrm{C}$ for times ranging from 3 to 15 min, followed by quenching below the glass transition temperature outside the calorimeter by immersion in liquid nitrogen with the maximum speed of transfer, and reheating at $20^{\circ} \mathrm{C} / \mathrm{min}$. The results are shown in Fig. 12: as can be seen, the data (full squares) concerning the samples kept at $260^{\circ} \mathrm{C}$ for the shortest times ( 3 and 5 min) lie between the solid line, calculated on the basis of the two phase-model, and 
the dashed one, which fits the experimental results obtained with the less drastic thermal treatments described in the Exptl. part $\left(t=1 \mathrm{~min}\right.$ at $260^{\circ} \mathrm{C}$ and partial melting). Therefore, it can be concluded that the samples subjected to these two thermal treatments are characterized by the presence of a rigid amorphous phase (even though their fraction is lower), analogous to the specimens described above. On the contrary, no rigid-amorphous phase is found if the polymer is kept at $260^{\circ} \mathrm{C}$ for longer times: in fact, the corresponding data fit well the $\Delta H_{\mathrm{m}} v s . \Delta c_{\mathrm{p}}$ curve calculated on the basis of the two-phase model (see full rhombi). In our opinion, under these experimental conditions, due to the low number of nuclei which persist in the melt, very few and large spherulites arise and consequently very few constraints of the crystal surface on the neighbouring amorphous phase result.

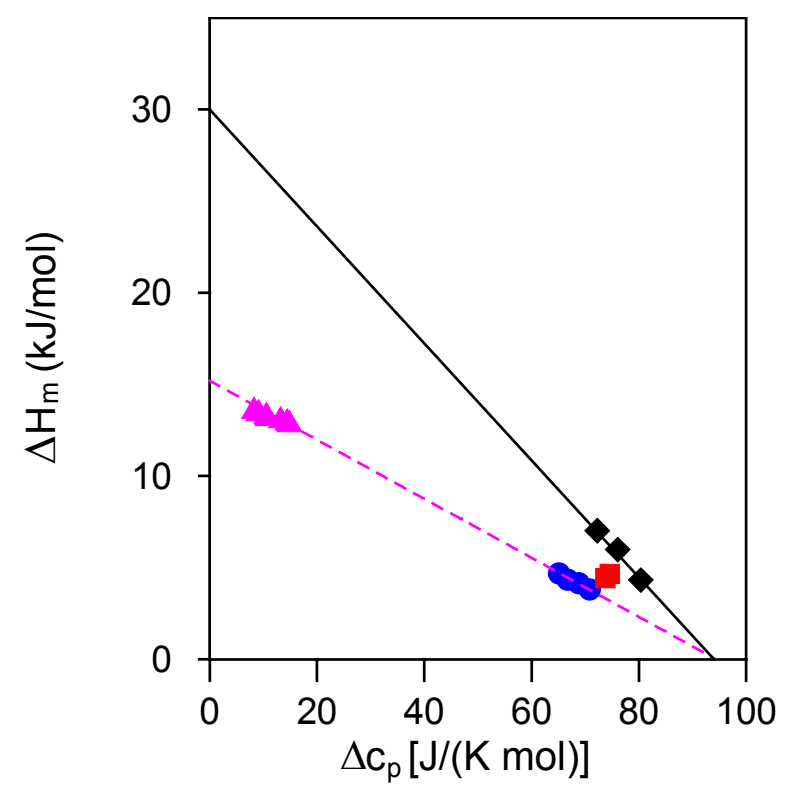

Fig. 12. Heat of fusion $\Delta H_{\mathrm{f}}$ as a function of the specific heat increment $\Delta c_{\mathrm{p}}$ at $T_{\mathrm{g}}$ :

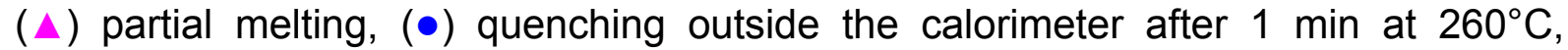
$(\bullet)$ quenching outside the calorimeter after 7,10 and $15 \mathrm{~min}$ at $260^{\circ} \mathrm{C},(\square)$ quenching outside the calorimeter after 3 and $5 \mathrm{~min}$ at $260^{\circ} \mathrm{C}$. The solid line was calculated on the basis of a two-phase model

\section{Conclusions}

Our investigations carried out on PPT led to some interesting results on the crystallization kinetics and melting behaviour:

The isothermal crystallization behaviour of PPT was found to be affected by the adopted thermal treatment, the overall crystallization rate regularly decreasing as the temperature and time of melting were increased. This trend can be explained on the basis of the well-known memory effect, which is correlated to residual athermal nuclei; the amount of these latter was found to decrease with increasing both fusion temperature and holding time. In all cases, independent of the adopted thermal treatment, a decrement of the overall crystallization rate with the crystallization temperature was observed, as expected at high values of $T_{\mathrm{c}}$ (low undercooling), where the determining step of the crystallization process is nucleation, the diffusion rate being high at high temperature. Space-filling spherulites were found to develop at all the crystallization temperatures investigated. 
The double melting peaks observed in PPT were explained as due to scanninginduced reorganisation processes occurring during heating in order to repack the preexisting lamellae into crystals of a higher stability. Furthermore, it was found that thermal lag could not be ignored and correction of the melting point to zero heating was necessary. The application of the non-linear Hoffman-Week treatment to PPT proved that a more reasonably and meaningfully estimation of $T_{\mathrm{m}}{ }^{\circ}$ can be obtained. In particular, it was demonstrated that the linear Hoffman-Week approach leads to a significant underestimation of the equilibrium melting temperature of PPT.

Finally, a rigid-amorphous phase was postulated, analogous to PBT and PET, which are the most important members of the series of engineering thermoplastic aromatic polyesters. The fraction of interphase was found to depend strongly on the adopted experimental conditions, being the lower the more drastic is thermal treatment. The observed trend was interpreted hypothesizing that less efficacious constraints of the crystal surface on the neighbouring amorphous phase arise for PPT samples characterized by a low density of nucleation and therefore few large spherulites.

[1] Turska, E.; Gogolewski, S.; J. Appl. Polym. Sci. 1975, 19, 637.

[2] Traub, H. L.; Angew. Makromol. Chem. 1995, 179, 4055.

[3] Bulkin, B. J.; Lewin, M.; Kim, J.; Macromolecules 1987, 20, 830.

[4] Chisholm, B. J.; Zimmer, J. G.; J. Appl. Polym. Sci. 2000, 76, 1296.

[5] Huang, J. M.; Chang, F. C.; J. Polym. Sci., Part B: Polym. Phys. 2000, 38, 934.

[6] Hong, P. D.; Chung, W. T.; Hsu, C. F.; Polymer 2002, 43, 3335.

[7] Toshihiko, J.; Shigeo, A.; Masao, S.; J. Macromol. Sci., Phys. B 1997, 36, 381.

[8] Cheng, S. Z. D.; Wu, Z. Q.; Wunderlich, B.; Macromolecules 1987, 20, 2802.

[9] Schick, C.; Wigger, J.; Mischok, W.; Acta Polym. 1990, 41, 137.

[10] Cheng, S. Z. D.; Pan, R.; Wunderlich, B.; Makromol. Chem. 1988, 189, 2443.

[11] Laredo, L.; Grimau, M.; Müller, A.; Bello, A.; Suarez, N.; J. Polym. Sci., Part B: Polym. Phys. 1996, 34, 2863.

[12] Huo, P.; Cebe, P.; Macromolecules 1992, 25, 902.

[13] Cheng, S. Z. D.; Cao, M. Y.; Wunderlich, B.; Macromolecules 1986, 19, 1868.

[14] Pyda, M.; Boller, A.; Grebowicz, J.; Chuah, H. H.; Lebedev, B. V.; Wunderlich, B.; J. Polym. Sci., Part B: Polym. Phys. 1998, 36, 2499.

[15] Pyda, M.; Wunderlich, B.; J. Polym. Sci., Part B: Polym. Phys. 2000, 38, 622.

[16] Grebowicz, J.; Chuah, H. H.; Progress Report, Shell Chemical Company, 1996.

[17] Hong, P. D.; Chuang, W. T.; Yeh, W. J.; Lin, T. L.; Polymer 2002, 43, 6879.

[18] Berti, C.; Bonora, V.; Colonna, M.; Lotti, N.; Sisti, L.; Eur. Polym. J. 2003, 39, 1595.

[19] Righetti, M. C.; Munari, A.; Macromol. Chem. Phys. 1997, 198, 363.

[20] Avrami, M.; J. Chem. Phys. 1941, 9, 177.

[21] Ziabicki, A.; Alfonso, G. C.; Colloid Polym. Sci. 1994, 272, 1027.

[22] Alfonso, G. C.; Ziabicki, A.; Colloid Polym. Sci. 1995, 273, 317. 
[23] Barham, P .J.; Keller, A.; Otun, E. L.; Holmes, P. A.; J. Mater. Sci. 1984, 19, 2781.

[24] Wu, P. L.; Woo, E. M.; J. Polym. Sci., Part B: Polym. Phys. 2002, 40, 1571.

[25] Jeong, Y. G.; Jo, W. H.; Lee, S. C.; Polymer 2003, 44, 3259.

[26] Holdsworth, P. J.; Turner-Jones, A.; Polymer 1971, 12, 195.

[27] Chung, J. S.; Cebe, P.; Polymer 1992, 33, 2312.

[28] Sohn, S.; Alizadeh, A.; Marand, H.; Polymer 2000, 41, 8879.

[29] Hoffman, J. D.; Weeks, J. J.; J. Res. Nat. Bur. Stand. 1962, 66A, 13.

[30] Xu, J.; Srinivas, S.; Marand, H.; Macromolecules 1998, 31, 8230.

[31] Marand, H.; Xu, J.; Srinivas, S.; Macromolecules 1998, 31, 8219.

[32] Hussein, M. A.; Strobl, G.; Macromolecules 2001, 34, 5.

[33] Hoffman, J. D.; Davis, G. T.; Lauritzen, J. I., Jr; in "Treatise on Solid State Chemistry", $1^{\text {st }}$ edition, Hannay, N. B., editor; vol. 3, chapter 7, Plenum Press, New York 1976.

[34] Suzie, P. D.; Perez, S.; Revol, J. F.; Brisse, F.; Polymer 1979, 20, 419.

[35] Thomas, D. G.; Stavely, L. A. K.; J. Chem. Soc. 1952, 4569.

[36] Cheng, S. Z. D.; Pan, R.; Bu, H. S.; Cao, M. Y.; Wunderlich, B.; Makromol. Chem. 1988, 189, 1579.

[37] Okazaki, I.; Wunderlich, B.; J. Polym. Sci., Part B: Polym. Phys. 1996, 34, 2941.

[38] Huo, P.; Cebe, P.; Colloid Polym. Sci. 1992, 270, 840. 